\title{
Low-temperature zero phonon lineshapes with various Brownian oscillator spectral densities
}

\author{
Robert S. Knox $^{\mathrm{a}, *}$, Gerald J. Small ${ }^{\mathrm{b}}$, Shaul Mukamel ${ }^{\mathrm{a}, \mathrm{c}}$ \\ a Department of Physics and Astronomy, University of Rochester, Rochester, NY 14627-0171, USA \\ ${ }^{\mathrm{b}}$ Department of Chemistry, Iowa State University, Ames, IA 50011-3111, USA \\ ${ }^{\mathrm{c}}$ Department of Chemistry, University of Rochester, Rochester, NY 14627-0216, USA
}

Received 29 January 2002

\begin{abstract}
The Brownian oscillator model has been successfully employed for modeling solvation dynamics in numerous femtosecond measurements. To a very large extent, this work has been interpreted on the basis of high-temperature limits of the theory. We present an analysis of the low temperature limit, which is particularly important for hole burning, photon echo, and single molecule spectroscopic experiments. Several forms for the bath spectral density are employed to compute zero phonon absorption line shapes. We show that in all cases the zero phonon linewidth vanishes at low temperatures, and that the line becomes asymmetric with a sharp rise at the red edge, as expected qualitatively. (c) 2002 Elsevier Science B.V. All rights reserved.
\end{abstract}

\section{Introduction}

The electron-phonon coupling and pure electronic dephasing of the $\mathrm{S}_{0} \rightarrow \mathrm{S}_{1}$ transition of molecules imbedded in host molecular solids at low temperatures (typically $\leqslant 20 \mathrm{~K}$ ) have long been subjects of considerable interest. The zero phonon line (ZPL) of the optical transition probes pure electronic dephasing. Linear electron-phonon coupling gives rise to phonon sideband structure associated with the ZPL [1]. This structure can be due to phonons of the host or pseudo-

\footnotetext{
${ }^{*}$ Corresponding author. Tel.: +1-716-275-8572; fax: +1-716275-8527.

E-mail address: rsk@pas.rochester.edu (R.S. Knox).
}

localized modes intimately associated with the guest molecule. In the former case the one-phonon profile is governed by the density of states of the host and a frequency dependent coupling constant. The latter has yet to be calculated for any molecular system. Within the context of the multimode Brownian oscillator (MBO) model [2], the pseudolocalized phonon would be a primary oscillator whose decay is governed by coupling to bath modes. Off-diagonal quadratic electron-phonon coupling, the analog of the molecular Duschinsky effect, is one mechanism for pure electronic dephasing [3]. Diagonal quadratic electron-phonon coupling associated with a pseudo-localized mode is another [4,5], the so-called exchange coupling mechanism. In organic systems comprised of large guest and host molecules the energies of phonons 
(intermolecular modes) that contribute to the phonon sideband are typically $\leqslant 50 \mathrm{~cm}^{-1}$. In this paper we are not concerned with higher energy intramolecular modes of the probe molecule.

During the 1970s absorption spectroscopy was used to study phonon structure and pure electronic dephasing in many mixed crystal systems (see, e.g., [6] and references therein). Later, photon echo and hole burning spectroscopies were brought to bear [7-10]. More complete theories of pure electronic dephasing emerged [11,12]. By the early 1980s our understanding of phonon structure and pure electronic dephasing had reached a rather mature stage. Most of the mixed crystal systems studied exhibited weak linear electron-phonon coupling (Huang-Rhys factor $\mathrm{S}<1$ ) which resulted in well resolved and symmetric ZPLs at sufficiently low temperatures. Attention then shifted to the homogeneous width of the ZPL in glasses and polymers. Initially, spectral hole burning was the technique used to probe the homogeneous width of the ZPL and its associated phonon structure $[13,14]$. The results, together with those of time-resolved resonant fluorescence line narrowing experiments on trivalent rare earth ions in inorganic glasses [1518], established that the magnitude and temperature dependence of the ZPL width are anomalous compared to those in crystals at low temperatures. The connection with the bistable tunneling configurations (two-level systems, TLS) responsible for the anomalous heat capacity, thermal conductivity and acoustic properties of glasses at very low temperatures [19] was quickly made [13,20-22]. Several theories for pure electronic dephasing emerged which explained the close to linear dependence of the ZPL width on temperature $(\leqslant 10 \mathrm{~K})$. For reviews see [23-25]. Following the above studies of the ZPL, photon-echo [26,27] and time-resolved hole burning experiments $[28,29]$ were brought to bear on the problem of the magnitude and temperature dependence of the ZPL profile. A key finding was that the homogeneous ZPL width in amorphous solids can depend on the "waiting" time between preparation of the coherent state and probing of the ZPL. This is a consequence of spectral diffusion due to tunneling of TLS that occurs on a longer time scale than the excited state lifetime. Again, the focus in the experiments with amorphous hosts was on chromophores exhibiting weak linear electron-phonon coupling. This coupling is typically dominated by a one-phonon profile with a peak energy of $\sim 15-30 \mathrm{~cm}^{-1}$. For sufficiently low burn fluences the zero phonon hole (ZPH) profiles were generally observed to be Lorentzian in high resolution experiments. Very recently, non-Lorentzian ZPH profiles were reported for Al-phthalocyanine tetrasulphonate in glassy water films [30]. These profiles were more sharply tipped than a Lorentzian but still symmetric. The non-Lorentzian behavior was shown to be a consequence of the dispersive kinetics of non-photochemical hole burning due to the angle between the laser polarization and transition dipole, off-resonant absorption of the ZPL and the distribution of non-photochemical hole burning rates due to structural disorder.

Single molecule spectroscopy has also been applied to chromophores exhibiting weak electron-phonon coupling in crystals and polymers at low temperatures [31-34]. In all cases we are aware of the single molecule line shapes did not exhibit significant asymmetry (see, e.g., [35]). We note that the effects of TLS tunneling on single molecule line shapes have been studied theoretically [36-38] and that complex multi-component line shapes can, in principle, arise when an absorber is strongly coupled to one or two nearby TLS with slow jump rates (slow modulation limit). When this is not the case, i.e. the absorber is weakly coupled to a "sea" of TLS, symmetric line shapes are expected.

Of particular relevance to this paper is that at sufficiently low temperatures the ZPL of the guest/ host systems characterized by weak linear electron-phonon coupling exhibit symmetric profiles whose widths are determined by longitudinal relaxation and pure electronic dephasing/spectral diffusion. Thus, the coupled phonons appear to be underdamped to an extent that precludes a significant contribution from them to the ZPL profile, especially on its high energy side. (By way of calibration, ZPL widths as determined by hole burning, photon echo and single molecule spectroscopies for ${ }^{1} \pi \pi^{*}$ states with ns lifetimes are $<1$ $\mathrm{cm}^{-1}$ for $T \leqslant 10 \mathrm{~K}$.) That this is not the case at high enough temperatures is due in part to the strong temperature dependences of pure electronic 
dephasing and the Franck-Condon factor of the ZPL (see, e.g., [39]). Unfortunately there is a dearth of high-resolution data on systems that exhibit strong linear electron-phonon coupling. However, such coupling is unrelated to damping of phonons that is governed by off-diagonal quadratic electron-phonon coupling in the case of a pesudo-localized mode. Thus, even in the case of strong linear coupling the modes could be strongly underdamped.

In this paper we explore the application of the MBO model to the optical spectra of chromophores in host solids at low temperatures. The MBO model has been used successfully in the high temperature limit to interpret room temperature data on optical coherence loss of chromophores in liquids due to solvation dynamics [40-44]. Application of the model to optical spectra requires the incorporation of other electronic dephasing mechanisms into the linear response function in addition to the MBO model. The line shape of the transition at the position of the ZPL in the MBO model is determined by $\gamma_{j}(\omega), \omega_{j}$, and $S_{j}$ which are the frequency dependent damping constant, frequency, and Huang-Rhys factor of the $j$ th primary BO. As noted by Toutounji et al. [45], it is only when the frequency dependence of $\gamma_{j}$ is properly taken into account that one can adequately describe the contribution from phonon dynamics to the ZPL profile. Often this dependence is neglected (ohmic dissipation); this is expected to lead to a maximum contribution from phonons to absorption in the ZPL region. Toutounji et al. did not investigate non-ohmic dissipation, choosing instead to eliminate the MBO ZPL from the linear response function and to replace it with a Lorentzian ZPL with a width governed by electronic dephasing. Their main result was a response function that accounts for electronic dephasing and modes exhibiting linear coupling or diagonal quadratic coupling. Presented here is an analysis of how ohmic and non-ohmic damping affect the absorption spectra of the MBO model at low temperatures for systems exhibiting linearly coupled primary oscillators that are underdamped. It reveals how a physically reasonable non-ohmic damping function, $\gamma_{j}(\omega)$, can lead to an MBO ZPL that has essentially zero linewidth at sufficiently low temperatures. Thus, by multiplying the linear response function by the response function for other mechanisms of electronic dephasing the MBO model can be used to simulate absorption (emission) spectra of chromophores in host media.

In Section 2 we briefly review the formal basis of the MBO model to establish the notation and the level of approximation. Section 3 demonstrates the intricacy of approaching the low- $T$ limit, which is a result of the fact that certain parts of series expansions must be summed completely. Section 4 turns to the numerical evaluation of lineshapes for ohmic and non-ohmic damping and Section 5 summarizes our results.

\section{The multimode Brownian oscillator model}

The multimode Brownian oscillator (MBO) model is based on the classic work of Lax [46] and Kubo [47], taking advantage of their proof that a second-order cumulant expansion is exact for treating the absorption lineshape of linearly coupled electron-phonon systems. The origin of its name is that for certain forms of the spectral density the model can be described by a few collective oscillators that satisfy a Langevin equation, providing an intuitive physical picture and a basis for analyzing molecular dynamics simulations [2]. The model assumes a two electronic-level system with some primary nuclear coordinates coupled linearly to it and to a harmonic bath:

$H=|g\rangle H_{\mathrm{g}}\langle g|+| e\rangle H_{\mathrm{e}}\langle e|+H^{\prime}$,

where

$$
\begin{aligned}
& H_{\mathrm{g}}=\sum_{j}\left[\frac{p_{j}^{2}}{2 m_{j}}+\frac{1}{2} m_{j} \omega_{j}^{2} q_{j}^{2}\right], \\
& H_{\mathrm{e}}=\hbar \omega_{\mathrm{eg}}^{0}+\sum_{j}\left[\frac{p_{j}^{2}}{2 m_{j}}+\frac{1}{2} m_{j} \omega_{j}^{2}\left(q_{j}+d_{j}\right)^{2}\right],
\end{aligned}
$$

and

$$
H^{\prime}=\sum_{n}\left[\frac{p_{n}^{2}}{2 m_{n}}+\frac{1}{2} m_{n} \omega_{n}^{2}\left(q_{n}-\sum_{j} \frac{c_{n j} q_{j}}{m_{n} \omega_{n}^{2}}\right)^{2}\right] .
$$


Here $p_{j}\left(p_{n}\right), q_{j}\left(q_{n}\right)$, and $m_{j}\left(m_{n}\right)$ represent the momentum, the coordinate, and the mass of the $j$ th ( $n$ th) nuclear mode of the primary (bath) oscillators, respectively. $d_{j}$ is the displacement for the $j$ th nuclear mode in the excited electronic state. $H^{\prime}$ describes the bath oscillators and their coupling to the primary oscillators with a coupling strength $c_{n j}$. The cross terms in $q_{j} q_{n}[48,49]$ are responsible for damping.

The purely electronic energy level separation is $\hbar \omega_{\mathrm{eg}}^{0}$ and the energy gap coordinate operator is defined as

$U=H_{\mathrm{e}}-H_{\mathrm{g}}-\hbar \omega_{\mathrm{eg}}^{0}$.

Hereafter a single primary oscillator is assumed. The subscript $j$ is retained in order to identify parameters belonging to this oscillator but it is understood that $j=1$.

Linear absorption lineshapes are computed by truncating the cumulant expansion of the response function at second order [2]. This is exact for the present model. The parameters of the Hamiltonian are transferred to a correlation function

$C_{j}(t)=-\frac{1}{2 \hbar^{2}}\left[\left\langle U(t) U(0) \rho_{\mathrm{g}}\right\rangle-\left\langle U(0) U(t) \rho_{\mathrm{g}}\right\rangle\right]$,

where $U(t)$ is the operator $U$ in the interaction representation and $\rho_{\mathrm{g}}$ is the ground-state vibrational density matrix. The Fourier transform of the correlation function has an imaginary part $[2$, Eqs. (8.64)] known as the spectral density:

$\tilde{C}_{j}^{\prime \prime}(\omega)=\frac{2 \lambda_{j} \omega_{j}^{2} \omega \gamma_{j}(\omega)}{\omega^{2} \gamma_{j}^{2}(\omega)+\left[\omega_{j}^{2}+\omega \Sigma_{j}(\omega)-\omega^{2}\right]^{2}}$.

Here $2 \lambda_{j}$ is the $j$ th mode contribution to the Stokes shift

$2 \lambda_{j}=\frac{m_{j} \omega_{j}^{2} d_{j}^{2}}{\hbar}$.

Alternatively we can write $\lambda_{j}=S_{j} \hbar \omega_{j}$, where $S_{j}$ is the dimensionless Huang-Rhys factor. $S_{j}$ can vary from much less than unity (weak coupling) to equal to greater than unity (moderate/strong coupling). The spectral distribution of the coupling is

$\gamma_{j}(\omega)=\frac{\pi}{m_{j}} \sum_{n} \frac{c_{n j}^{2}}{2 m_{n} \omega_{n}^{2}}\left[\delta\left(\omega-\omega_{n}\right)+\delta\left(\omega+\omega_{n}\right)\right]$.
The real part $\Sigma_{j}(\omega)$ of the self-energy is related to $\gamma_{i}(\omega)$ by the Kramers-Kronig relation [16]:

$\Sigma_{j}(\omega)=-\frac{1}{\pi} \mathscr{P} \mathscr{P} \int_{-\infty}^{\infty} \mathrm{d} \omega^{\prime} \frac{\gamma_{j}\left(\omega^{\prime}\right)}{\omega^{\prime}-\omega}$,

where $\mathscr{P} \mathscr{P}$ stands for principal part.The absorption lineshape is given by

$\sigma_{\mathrm{BO}}(\omega)=\frac{1}{\pi} \operatorname{Re} \int_{0}^{\infty} \mathrm{d} t \exp \left[\mathrm{i}\left(\omega-\omega_{\mathrm{eg}}^{0}-\lambda_{j}\right) t-g(t)\right]$,

where [2, Eq. (8.13a)]

$g_{j}(t)=\int_{0}^{t} \mathrm{~d} \tau_{1} \int_{0}^{\tau_{1}} \mathrm{~d} \tau_{2} C_{j}\left(\tau_{2}\right)$,

or, in the Fourier representation [2, Eq. (8.25)],

$$
\begin{aligned}
g_{j}(t)= & -\frac{1}{2 \pi} \int_{-\infty}^{\infty} \mathrm{d} \omega \frac{\tilde{C}_{j}^{\prime \prime}(\omega)}{\omega^{2}} \\
& \times[1+\operatorname{coth}(\beta \hbar \omega / 2)]\left[\mathrm{e}^{-\mathrm{i} \omega t}+\mathrm{i} \omega t-1\right] .
\end{aligned}
$$

The Boltzmann factor $\operatorname{coth}(\beta \hbar \omega / 2)$, where $\beta=1 / k_{\mathrm{B}} T$, reflects the thermal population of the bath modes.

\section{Ohmic dissipation; linewidth at low temperature}

When $\gamma_{j} \gg \omega_{j}$ the primary oscillator is overdamped and causes line broadening. In this paper we focus on the opposite underdamped limit $\omega_{j} \gg \gamma_{j}$ where a progression of primary oscillator vibrational lines appears. At zero temperature we expect the ZPL linewidth to vanish. The way it does depends on the spectral density. In the absence of detailed information about the bath and its coupling, the damping $\gamma_{i}(\omega)$ is often approximated by a constant, $\gamma_{j}$. This simplification, known as the ohmic approximation, is made largely for convenience, because the physical meaning would be that the product of mode density and interaction strength is a constant, for which there is no formal justification. Should the approximation happen to be a good one, however, it simplifies the theory in that it enables certain analytic expressions to be derived and the self-energy correction equation (10) vanishes. In the numerical 
work presented below we omit self energies altogether, regardless of the spectral dependence of the damping, because our interest centers on lineshapes.

We write $C_{j}(t)$ in terms of its real and imaginary parts $C_{j}^{\prime}(t)$ and $C_{j}^{\prime \prime}(t)$, respectively:

$C_{j}(t)=C_{j}^{\prime}(t)+\mathrm{i} C_{j}^{\prime \prime}(t)$

In the ohmic case these functions are given by [2, Eqs. (8.67a)-(8.67c)]

$$
\begin{aligned}
C_{j}^{\prime}(t)= & \frac{\hbar}{4 m_{j} \zeta_{j}}\left[\operatorname{coth}\left(\mathrm{i} \phi_{j} \hbar \beta / 2\right) \exp \left(-\phi_{j} t\right)\right. \\
& \left.+\operatorname{coth}\left(\mathrm{i} \phi_{j}^{\prime} \hbar \beta / 2\right) \exp \left(-\phi_{j}^{\prime} t\right)\right] \\
& -\frac{2 \gamma_{j}}{m_{j} \beta} \sum_{n=1}^{\infty} \frac{v_{n} \exp \left(-v_{n} t\right)}{\left(\omega_{j}^{2}+v_{n}^{2}\right)^{2}-\gamma_{j}^{2} v_{n}^{2}}
\end{aligned}
$$

and

$$
C_{j}^{\prime \prime}(t)=-\frac{\hbar}{2 m_{j}} \cdot \frac{1}{\zeta_{j}} \exp \left(-\gamma_{j}|t| / 2\right) \sin \left(\zeta_{j} t\right) .
$$

Here $\quad \zeta_{j}=\left(\omega_{j}^{2}-\gamma_{j}^{2}\right)^{1 / 4}, \quad \phi_{j}=\gamma_{j} / 2+\mathrm{i} \zeta_{j}, \quad \phi_{j}^{\prime}=$ $\gamma_{j} / 2-\mathrm{i} \zeta_{j}$, and $v_{n}=(2 \pi / \hbar \beta) n$ (the Matsubara frequencies).

The absorption spectrum associated with the MBO model for a single primary oscillator consists of a ZPL and a series of satellites at $\hbar \omega_{\mathrm{eg}}^{0} \pm n \hbar \omega_{j}(n=1,2, \ldots)$. The widths and relative strengths of all the lines depend on the parameter $S_{j}$, the temperature $T$, and the magnitude and shape of the damping $\gamma_{i}(\omega)$. At low temperature, we expect that the ZPL for this model will have zero width regardless of the precise frequency dependence of the damping, because phononic relaxation is absent and electronic dephasing has not been included.

Let us consider the low-temperature expression for $g_{j}(t): C_{j}^{\prime \prime}(t)$ does not depend on temperature, and for $C_{j}^{\prime}(t)$ we have in this limit

$C_{j}^{\prime}(t)=\Delta_{j}^{2} \exp \left(-\phi_{j} t\right)-\frac{2 \gamma_{j}}{m_{j} \beta} \sum_{n=1}^{\infty} \frac{v_{n} \exp \left(-v_{n} t\right)}{\left(\omega_{j}^{2}+v_{n}^{2}\right)^{2}-\gamma_{j}^{2} v_{n}^{2}}$.

We have defined $\Delta_{j}^{2}=\omega_{j}^{3} \delta_{j}^{2} / 2 \zeta_{j}$, where $\delta_{j}=$ $\left(m_{j} \omega_{j} / h\right)^{1 / 2} d_{j}$ is the dimensionless displacement parameter. An oscillator $j$ coupled to an electronic system makes the following contribution to the function $g_{j}(t)[15$, p. 229, Eq. (8.67a)]:

$g_{j}(t)=g_{j}^{\prime}(t)+g_{j}^{\prime \prime}(t)$,

where $g_{j}^{\prime \prime}(t)$ is purely imaginary in the present application (low damping, defined as $\gamma_{j} \ll 2 \omega_{j}$ ). Using Eq. (17) we get

$g_{j}^{\prime}(t)=g_{j}^{\mathrm{I}}(t)+g_{j}^{\mathrm{II}}(t)$,

where

$g_{j}^{\mathrm{I}}(t)=\frac{\Delta_{j}^{2}}{\phi_{j}^{2}}\left[\phi_{j} t+\exp \left(-\phi_{j} t\right)-1\right]$

and the contribution of the Matsubara series is

$g_{j}^{\mathrm{II}}(t)=-\frac{2 \gamma_{j} \xi_{j}^{2}}{m_{j} \beta} \sum_{n=1}^{\infty} \frac{v_{n} \int_{0}^{t} \mathrm{~d} \tau_{1} \int_{0}^{\tau_{1}} \mathrm{~d} \tau_{2} \exp \left(-v_{n} \tau_{2}\right)}{\left(\omega_{j}^{2}+v_{n}^{2}\right)^{2}-\gamma_{j}^{2} v_{n}^{2}}$.

Performing the double integral in the numerator we obtain

$g_{j}^{\mathrm{II}}(t)=-\frac{4 \Delta_{j}^{2} \gamma_{j} \zeta_{j}}{\hbar \beta} \sum_{n=1}^{\infty} \frac{t-v_{n}^{-1}\left(1-\exp \left(-v_{n} t\right)\right)}{\left(\omega_{j}^{2}+v_{n}^{2}\right)^{2}-\gamma_{j}^{2} v_{n}^{2}}$.

Summing this series in the low-temperature limit can be accomplished by making use of the fact that large- $n$ (thus large $v_{n}$ ) terms are dominant for large $\beta$, which allows the sum to be well approximated by an integral. The general results are rather complicated in form, but it is possible to extract the contribution of the sum to $g_{j}^{\mathrm{II}}(t)$ that is linear in $t$, and it precisely cancels the linear term appearing in $g_{j}^{\mathrm{I}}(t)$. For the term in Eq. (22) that is linear in $t$, we make the approximation

$$
\begin{aligned}
& \sum_{n=1}^{\infty} \frac{1}{\left(\omega_{j}^{2}+v_{n}^{2}\right)^{2}-\gamma_{j}^{2} v_{n}^{2}} \\
& \quad=\sum_{n=1}^{\infty} \frac{1}{\left(\omega_{j}^{2}+T_{a}^{2} n^{2}\right)^{2}-\gamma_{j}^{2} T_{a}^{2} n^{2}} \\
& \quad \sim \frac{1}{T_{a}} \int_{0}^{\infty} \frac{\mathrm{d} x}{\left(\omega_{j}^{2}+x^{2}\right)^{2}-\gamma_{j}^{2} x^{2}}
\end{aligned}
$$


where $x=v_{n}=T_{a} n$ and $T_{a}=2 \pi / \hbar \beta$ (temperature in angular frequency units). Eq. (23) is readily integrated, giving

$$
\begin{aligned}
\frac{1}{2 \mathrm{i} \gamma_{j} \zeta_{j} T_{a}}\left[\frac{\tan ^{-1}\left(x / \sqrt{\Omega_{j}^{2}-\mathrm{i} \gamma_{j} \zeta_{j}}\right)}{\sqrt{\Omega_{j}^{2}-\mathrm{i} \gamma_{j} \zeta_{j}}}\right. \\
\left.-\frac{\tan ^{-1}\left(x / \sqrt{\Omega_{j}^{2}+\mathrm{i} \gamma_{j} \zeta_{j}}\right)}{\sqrt{\Omega_{j}^{2}+\mathrm{i} \gamma_{j} \zeta_{j}}}\right]_{0}^{\infty},
\end{aligned}
$$

where $\Omega_{j}=\left(\omega_{j}^{2}-\gamma_{j}^{2} / 2\right)^{1 / 2}$. This expression vanishes at the lower limit, leaving

$$
\begin{aligned}
& \frac{\pi}{4 \mathrm{i} \gamma_{j} \zeta_{j} T_{a}}\left(\frac{1}{\sqrt{\Omega_{j}^{2}-\mathrm{i} \gamma_{j} \zeta_{j}}}-\frac{1}{\sqrt{\Omega_{j}^{2}+\mathrm{i} \gamma_{j} \zeta_{j}}}\right) \\
& =\frac{\pi}{4 \mathrm{i} \gamma_{j} \zeta_{j} \omega_{j}^{2} T_{a}} \mathrm{i} \gamma_{j}=\frac{\pi}{4 \zeta_{j} \omega \omega_{j}^{2} T_{a}} .
\end{aligned}
$$

Reduction to such a simple form is greatly facilitated by the fact that $\left(\Omega_{j}^{4}-\gamma_{j}^{2} \zeta_{j}^{2}\right)^{1 / 2}=\omega_{j}^{2}$. When expression (25) is used to evaluate the term of Eq. (22) that is linear in $t$, we find

$$
\begin{aligned}
\frac{\left[g_{j}^{\mathrm{II}}(t)\right]_{\text {lin }}}{t} & \sim \frac{4 \Delta_{j}^{2} \gamma_{j} \zeta_{j}}{\hbar \beta} \cdot \frac{\pi}{4 \zeta_{j} \omega_{j}^{2} T_{a}}=-\frac{4 \Delta_{j}^{2} \gamma_{j}}{8 \omega_{j}^{2}} \\
& =-\frac{\omega_{j} \gamma_{j} d_{j}^{2}}{4 \zeta_{j}} .
\end{aligned}
$$

This term will exactly cancel the real part of the linear term in $g_{j}^{\mathrm{I}}(t)$ :

$$
\begin{aligned}
\frac{\left[g_{j}^{\mathrm{I}}(t)\right]_{\mathrm{lin}}}{t} & \sim \frac{\Delta_{j}^{2}}{\phi_{j}}=\frac{\omega_{j}^{3} d_{j}^{2}}{2 \zeta_{j}} \cdot \frac{1}{\frac{1}{2} \gamma_{j}+\mathrm{i} \zeta_{j}} \\
& =\frac{\omega_{j} d_{j}^{2}}{2 \zeta_{j}}\left(\frac{1}{2} \gamma_{j}-\mathrm{i} \zeta_{j}\right) .
\end{aligned}
$$

The remaining sums in $g_{j}^{\mathrm{II}}(t)$ consist of a shortlived damping factor and a constant.

In our numerical evaluation of the equivalent expression (13) for $g_{j}(t)$, we have found that the real part of $g_{j}(t)$ is finite at large $t$ and contains no term linear in $t$, as shown in Fig. 1.

If we neglect $g_{j}^{\mathrm{II}}(t)$ the system response function has the form

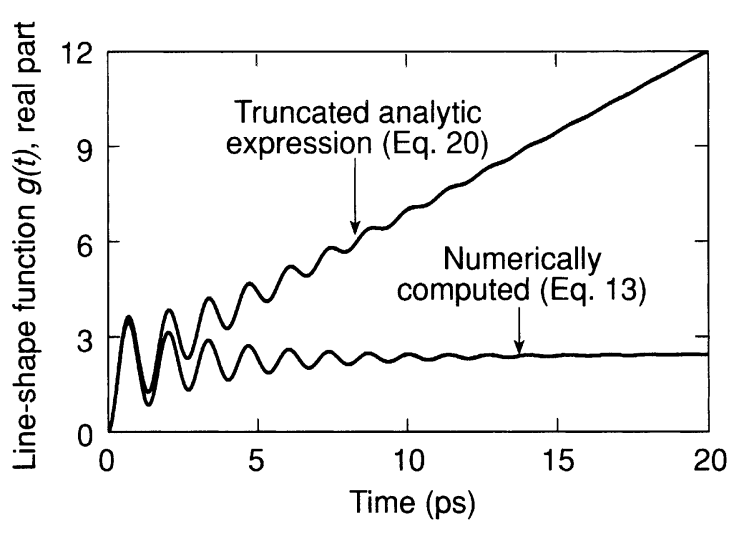

Fig. 1. Comparison of the real part of the line shape function $g_{j}(t)$ obtained analytically by truncating the correlation function (upper curve) and numerically (lower curve). The parameters used in each case $\gamma_{j}=3 \mathrm{~cm}^{-1}, \omega_{j}=25 \mathrm{~cm}^{-1}, S_{j}=1.8$, and $T=0.01 \mathrm{~K}$.

$$
\begin{aligned}
J(\omega)= & -\mathrm{i} \int_{0}^{\infty} \mathrm{d} t \exp \left[\mathrm{i} \omega t-g_{j}^{\mathrm{I}}(t)\right] \\
= & A \sum_{n=0}^{\infty} \frac{1}{n !}\left(-\Delta_{j}^{2} / \phi_{j}^{2}\right)^{n} \\
& \times \int_{0}^{\infty} \mathrm{d} t \exp \left\{\left[\left(\mathrm{i} \omega-n \phi_{j}-\left(\Delta_{j}^{2} / \phi_{j}\right)\right] t\right\},\right.
\end{aligned}
$$

where $A$ absorbs non-essential factors and the summation comes from an expansion of the middle term of Eq. (20) in a Taylor series. The integral, including a factor $-\mathrm{i}$ for convenience, has the lowdamping limit value

$\frac{1}{\omega+\mathrm{i}\left(n \phi_{j}+\frac{\Delta_{j}^{2}}{\phi_{j}}\right)} \gamma_{j} \ll \omega_{j} \frac{1}{\omega-n \omega_{j}+S_{j} \omega_{j}+\mathrm{i} \gamma_{j}\left(\frac{n+S_{j}}{2}\right)}$.

(We have used the fact that $S_{j}=\Delta_{j}^{2} / \omega_{j}^{2}$ in this limit.) Therefore, under these conditions the predicted partial width of the ZPL $(n=0)$ is $2 S_{j} \gamma_{j}$. Furthermore, this width is directly traceable to the linear term of (20) via the last term in the square brackets in Eq. (28). This implies that in the case of low ohmic damping strength $\left(\gamma_{j} \ll \omega_{j}\right)$ the lowtemperature width of lines in the MBO spectrum would be $2 S_{j}\left(2 n_{j}+1\right) \gamma_{j}$, where $n_{j}$ is the thermal occupation number of oscillator $j$, and therefore approach a finite value $2 S_{j} \gamma_{j}$ as $T \rightarrow 0$ [14]. However 
once the Matsubara terms are included this width vanishes.

Fig. 1 shows a comparison of the function $g_{j}^{\mathrm{I}}(t)$, Eq. (20), with a direct numerical evaluation of $g_{j}^{\prime}(t)$ from the real part of Eq. (13). The large secular increase in $g_{j}^{\mathrm{I}}(t)$ is responsible for the excess width. The imaginary part $g_{j}^{\prime \prime}(t)$ is reproduced rather precisely by numerical calculations.

\section{Lineshapes for ohmic and non-ohmic damping profiles}

To model damping strengths with spectral structure, we adopt the form

$\gamma_{j s}(\omega)=\gamma_{j 0} \cdot \omega^{s} \cdot \exp \left(-\omega / \omega_{\mathrm{D}}\right)$,

which gives a maximal broadening of the primary oscillator structure for sidebands that are dis- placed by an amount $s \omega_{\mathrm{D}}$ from the zero point line. The temperature dependence of the MBO ZPL and nearby lines is studied by applying the numerical method employed by Zhao and Knox [50], which consists of trapezoidal integration to obtain the functions leading up to the absorption line shape. After appropriate parameters and a functional form of $\gamma_{j}(\omega)$ is chosen, $C_{j}^{\prime \prime}(\omega)$ is obtained from Eq. (7). The grid of $\omega$ values for the subsequent integration is checked by testing the numerical value of the integral

$\lambda_{j}=\frac{1}{\pi} \int_{0}^{\infty} \mathrm{d} \omega \frac{C_{j}^{\prime \prime}(\omega)}{\omega}$,

$g_{j}(t)$ is then obtained from Eq. (13), and finally $\sigma_{\mathrm{BO}}(\omega)$ is calculated from Eq. (11). The time integration grid is checked by varying $t_{\max }$ (the alias of infinity), and the number $n_{t}$ of grid points, which is typically 20,000 or 40,000 for $t_{\max }=200$ ps. The
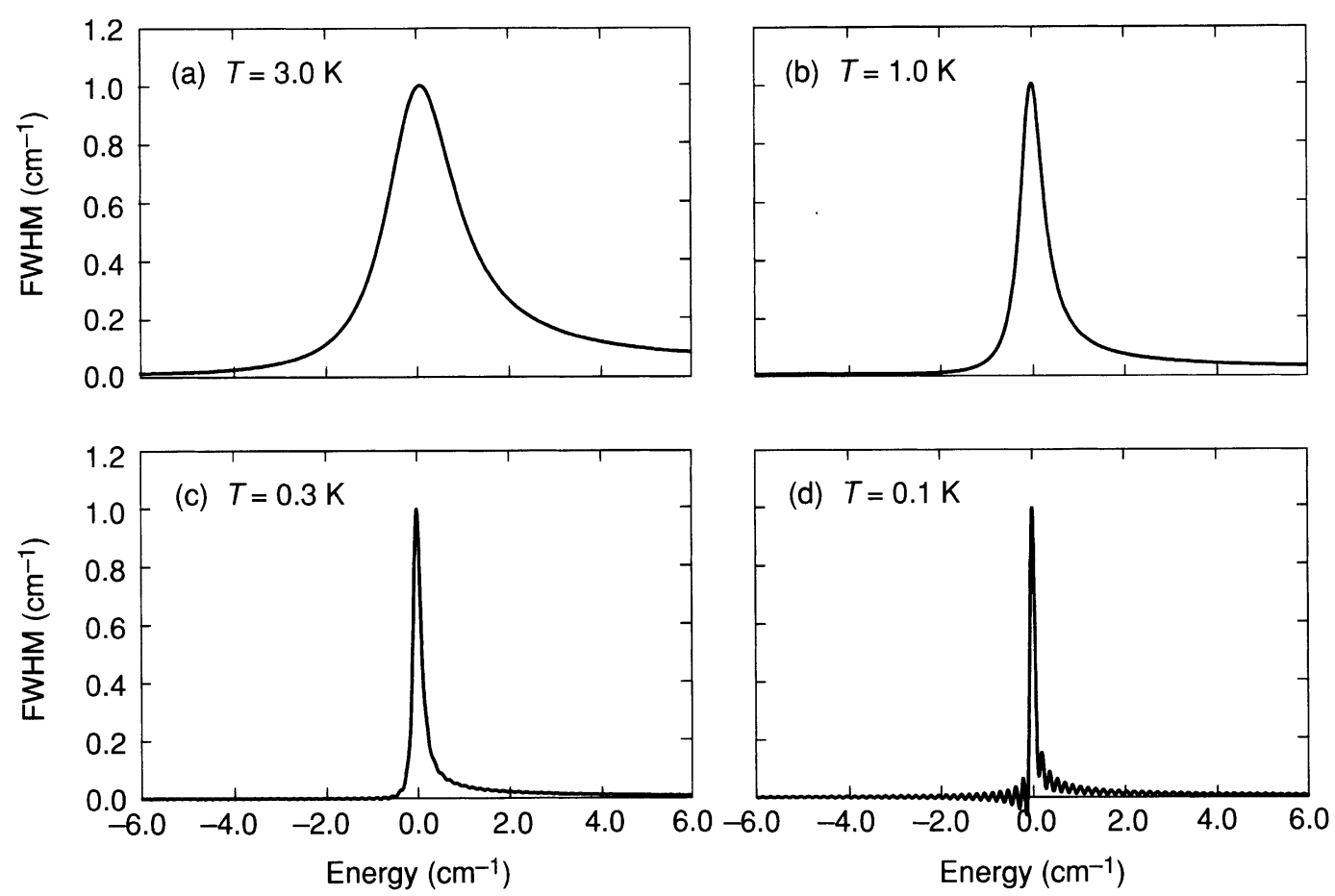

Fig. 2. Narrowing of the zero phonon line (ZPL) with decreasing temperature in a multimode Brownian oscillator (BO) model absorption spectrum in the low-ohmic-damping limit. Parameters are $\gamma_{j}=3 \mathrm{~cm}^{-1}, \omega_{j}=25 \mathrm{~cm}^{-1}$, and $S_{j}=1.8$. The four temperatures correspond to $0.07,0.21,0.70$, and $2.1 \mathrm{~cm}^{-1}$. Spectra are scaled to unity at maximum. The original relative peak heights were 12.55:6.78:2.55:1. Energy is measured relative to the $0-0$ transition energy, and no self-energy corrections have been made. Oscillations in the $T=0.1$ and $0.3 \mathrm{~K}$ cases are numerical artifacts imposed by the Fourier transform time range. 
final integral being a Fourier transform, a limiting accuracy of $\sim n_{t} / t_{\max }$ is placed on the frequency (energy) dependence of the result. In order to remind the reader of this limitation, we have not smoothed any resulting spurious oscillations. In the following examples, all frequencies and energies are expressed as wavenumbers and all spectra are plotted on a scale whose zero corresponds to $\hbar \omega_{\mathrm{eg}}^{0}$.

Fig. 2 shows spectra for an ohmic damping of $\gamma_{j}=3 \mathrm{~cm}^{-1}$ and $S_{j}=1.8$, illustrating the fact that the ZPL has a width much narrower than $2 S_{j} \gamma_{j}=10.8 \mathrm{~cm}^{-1}$. There is a clear asymmetry of the ZPL arising from the lack of occupied bath modes at low temperature. Phonon absorption processes are impossible and hence a sharply dropping low-energy profile results on the ZPL. Conversely, bath phonon emission processes can occur and the high-energy profile is not suppressed.
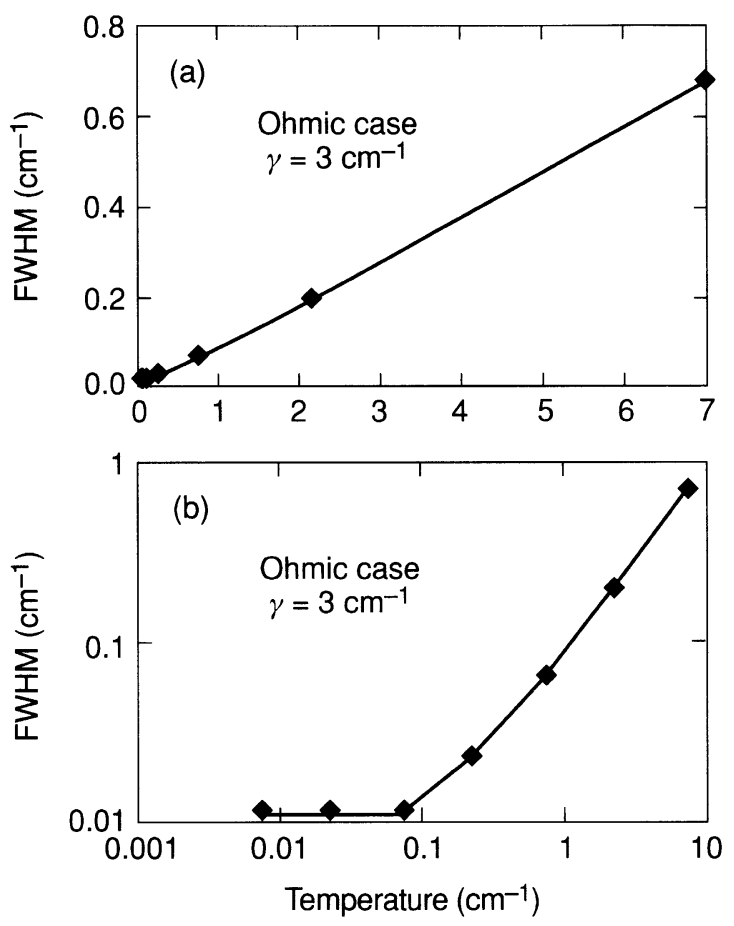

Fig. 3. (a) Computed ZPL width for the case $\gamma_{j}=3 \mathrm{~cm}^{-1}$, $\omega_{j}=25 \mathrm{~cm}^{-1}$, and $S_{j}=1.8$. The width is clearly approaching zero with temperature. (b) Log plot of the data, showing the limit imposed by numerical methods at $T<0.1 \mathrm{~cm}^{-1}$.
In Fig. 3(a) and in Fig. 5 the ZPL width is seen to rise essentially linearly with temperature. This happens because the phonon occupation number is the only variable or parameter determining the width, under the white-noise conditions of ohmic damping. Fig. 3(b) repeats (a) in log scale, magnifying the low-temperature portion. The effect of the limited accuracy of the simplified Fourier transform algorithm is clearly seen. It is also manifested in the oscillations in Fig. 2 at $0.1 \mathrm{~K}$, where an attempt is being made to approximate a near-delta function.

When ohmic damping is replaced by Eq. (30), the linewidth picture changes substantially. First we illustrate the situation at $T=30 \mathrm{~K}$, Fig. 4 . As the parameter $s$ increases from 0.25 to 0.75 , the ZPL narrows greatly. This can be attributed to the fact that the coupling to bath modes in the vicinity of zero frequency is increasingly suppressed as $s$ rises. The $n= \pm 1$ lines at $\omega=$ $\pm 25 \mathrm{~cm}^{-1}$ remain at a fairly constant width be-

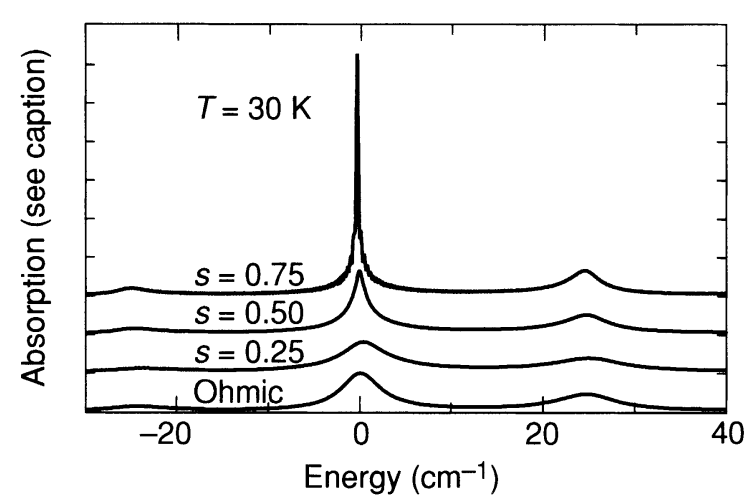

Fig. 4. Effect of damping function on spectra at $30 \mathrm{~K}$ for ohmic damping and non-ohmic damping of the form $\gamma_{j}(\omega)=$ $\gamma_{j s} \omega^{s} \exp \left(-\omega / \omega_{\mathrm{D}}\right)$ for $s=0.25,0.50$, and 0.75 . Frequency parameters are $\omega_{\mathrm{D}}=\omega=25 \mathrm{~cm}^{-1}$. In these examples, $S_{j}=0.5$. The damping strength $\gamma_{j s}$ has been chosen in each non-ohmic case to make $\gamma_{j}\left(\omega_{j}\right)=3 \mathrm{~cm}^{-1}$, which is the value of the constant $\gamma_{j}$ in the ohmic case. This maintains a fairly constant $n=1$ linewidth as $s$ varies. Curves are displaced vertically to enable comparison of line shapes, but the vertical scale is otherwise the same for all four cases. As $s$ increases, the lowest-frequency damping $\gamma_{j}(\omega \sim 0)$ is better suppressed and a sharp line increasingly dominates the $0-0$ transition region. Again energy is measured relative to the $0-0$ transition energy, and no selfenergy corrections have been made. 

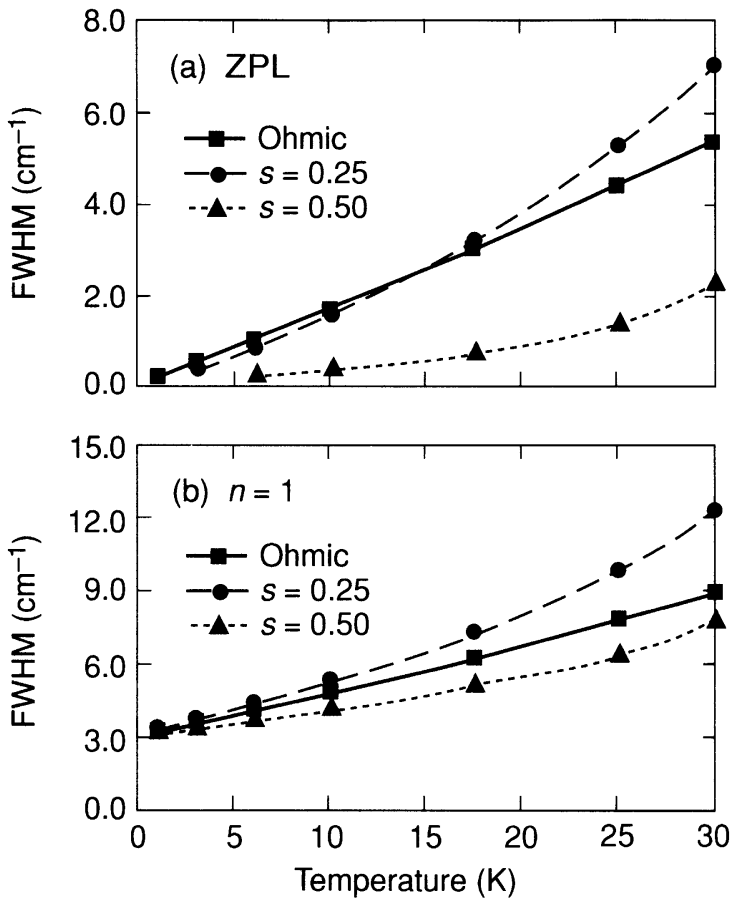

Fig. 5. Temperature dependences of the widths of the ZPL and the $n=1$ line for ohmic damping and non-ohmic damping of the form $\gamma_{j}(\omega)=\gamma_{j s} \omega^{s} \exp \left(-\omega / \omega_{\mathrm{D}}\right)$ for $s=0.25$ and 0.50 . Other parameters are chosen as described in the caption of Fig. 4 and with the same values.

cause we have deliberately chosen the damping function (30) to have the value $3 \mathrm{~cm}^{-1}$ at $\omega=25 \mathrm{~cm}^{-1}$ for each value of $s$.

Fig. 5 shows the temperature dependence of the widths of the zero phonon and $n=1$ lines. The depression of the ZPL width at both $s=0.25$ and $s=0.50$ is clear, although in the $s=0.25$ case the ZPL width depression is restricted to lower temperatures. In that case, $(s=0.25)$ the damping maximizes at $6.25 \mathrm{~cm}^{-1}$, and its value at that energy is relatively large $\left(4.49 \mathrm{~cm}^{-1}\right)$ because of the requirement that $\gamma_{j s}(25)=3$. The $n=1$ linewidth at low temperature is independent of $s$ because of this same arbitrary requirement. Note the rather good linearity of the ZPL width in the ohmic case. The numerical calculations show that here the term in $g_{j}^{\prime}(t)$ that is linear in time is also linear in temperature in the temperature range shown, a fact which might be directly related to the linearity in width.
The parameter $s$ that characterizes non-ohmic damping in our calculations (see Eq. (30)) should be equal to or greater than 2 in practice. A value $s=2$ corresponds to a Debye density of states for low frequency bath modes. A primary oscillator is likely to be librational in nature. Thus, it should couple weakly to low frequency acoustic modes of the bath which would increase $s$ above 2. Additional calculations (not shown) were performed with larger values of $s$, particularly 2 and 4 . All linewidths were completely suppressed at low temperatures and the numerical calculation became unstable as $T$ was increased.

\section{Concluding remarks}

In this paper we analyze the MBO lineshape in the low temperature limit. On physical grounds we expect the ZPL lineshape to become asymmetric as $T \rightarrow 0$ with a sharp rise in the low frequency edge since the bath can only accept, rather than provide, energy in this case. We further expect the linewidth to vanish at $T=0$ where the bath fluctuations are suppressed. Our calculations demonstrate these trends analytically in the case of ohmic damping and numerically for certain representative non-ohmic spectral densities. The variation of the linewidth with temperature depends strongly on the precise form of the spectral density, as illustrated in our calculations for the non-ohmic dissipation model. For ohmic dissipation, the vanishing of the linewidth involves a delicate cancellation of terms involving the series of Matsubara frequencies. The Matsubara expansion is very useful at high temperatures where it converges rapidly. At low temperatures, the entire series (Eq. (15)) needs to be resummed and it may be better to abandon the expansion altogether and directly carry out the Fourier expansion (Eq. (13)) numerically. Since the MBO linewidth vanishes at $T=0$, we expect lifetime broadening to take over and dominate the lineshape. However, at finite and low temperatures the MBO model may make a significant contribution to the linewidth, in addition to other electronic dephasing mechanisms such as coupling to a bath made of two level systems in glasses. 


\section{Acknowledgements}

GJS thanks the Solid State Chemistry and Polymers Program of the NSF for support under grant no. DMR-9908714. SM gratefully acknowledges the support of the National Science Foundation and the Petroleum Research Fund administered by the American Chemical Society.

\section{References}

[1] K.K. Rebane, Impurity Spectra of Solids: Elementary Theory of Vibrational Structure, Plenum Press, New York, 1970.

[2] S. Mukamel, Principles of Nonlinear Optical Spectroscopy, Oxford University Press, Oxford, 1995.

[3] D.E. McCumber, M.D. Sturge, J. Appl. Phys. 34 (1963) 1682.

[4] R.M. Shelby, C.B. Harris, P.A. Cornelius, J. Chem. Phys. 70 (1979) 34.

[5] B. Jackson, R. Silbey, Chem. Phys. Lett. 99 (1979) 331.

[6] F.P. Burke, G.J. Small, Chem. Phys. 5 (1974) 198.

[7] W.H. Hesselink, D.A. Wiersma, in: V.M. Agranovich, R.M. Hochstrasser (Eds.), Spectroscopy and Excitation Dynamics of Condensed Molecular Systems, North-Holland, Amsterdam, 1983, p. 249 (Chapter 6).

[8] M.J. Burns, W.K. Liu, A.H. Zewail, in: V.M. Agranovich, R.M. Hochstrasser (Eds.), Spectroscopy and Excitation Dynamics of Condensed Molecular Systems, North-Holland, Amsterdam, 1983, p. 301.

[9] K.K. Rebane, L.A. Rebane, in: W.E. Moerner (Ed.), Persistent Spectral Hole Burning: Science and Applications, Springer, Berlin, 1987, p. 17.

[10] S. Völker, R.M. Macfarlane, J.H. van der Waals, Chem. Phys. Lett. 53 (1978) 8.

[11] J.L. Skinner, Ann. Rev. Phys. Chem. 39 (1988) 463.

[12] I.S. Osad'ko, in: V.M. Agranovich, R.M. Hochstrasser (Eds.), Spectroscopy and Excitation Dynamics of Condensed Molecular Systems, North-Holland, Amsterdam, 1983, p. 437.

[13] G.J. Small, in: V.M. Agranovich, R.M. Hochstrasser (Eds.), Spectroscopy and Excitation Dynamics of Condensed Molecular Systems, North-Holland, Amsterdam, 1983 , p. 515 .

[14] W.E. Moerner (Ed.), Persistent Spectral Hole Burning: Science and Applications, Springer, Berlin, 1987.

[15] P.M. Selzer, D.L. Huber, D.S. Hamilton, W.M. Yen, M.J. Weber, Phys. Rev. Lett. 36 (1976) 813.

[16] P. Avouris, A. Campion, M.A. El-Sayed, J. Chem. Phys. 67 (1977) 3397.

[17] J.R. Morgan, M.A. El-Sayed, Chem. Phys. Lett. 84 (1981) 213.

[18] J. Hegarty, W.M. Yen, Phys. Rev. Lett. 43 (1979) 1126.
[19] W.A. Phillips (Ed.), Amorphous Solids: Low Temperature Properties, Springer, New York, 1981.

[20] J.M. Hayes, G.J. Small, Chem. Phys. 27 (1978) 151.

[21] S.K. Lyo, R. Orbach, Phys. Rev. B 22 (1980) 4223.

[22] T.L. Reinecke, K.L. Ngai, Phys. Rev. B 12 (1975) 3476.

[23] S. Völker, in: J. Fünfschilling (Ed.), Radiation Processes in Molecular Excited States, Kluwer Academic Publishers, Dordrecht, 1989, p. 113.

[24] J.M. Hayes, R. Jankowiak, G.J. Small, in: W.E. Moerner (Ed.), Persistent Spectral Hole Burning: Science and Applications, Springer, Berlin, 1987, p. 153.

[25] R. Silbey, K. Kassner, J. Lumin. 36 (1987) 283.

[26] L.R. Narasimhan, K.A. Littau, D.W. Pack, Y.S. Bai, A. Elschner, M.D. Fayer, Chem. Rev. 90 (1990) 439.

[27] J.B.W. Morsink, W.H. Hesselink, D.A. Wiersma, Chem. Phys. 71 (1992) 289.

[28] K.A. Littau, M.D. Fayer, Chem. Phys. Lett. 176 (1991) 551.

[29] P.J. van der Zaag, J.P. Galaup, S. Völker, Chem. Phys. Lett. 166 (1990) 263.

[30] T. Reinot, G.J. Small, J. Chem. Phys. 114 (2001) 9105.

[31] R. Rigler, P.G. Wolynes (Eds.), Chem. Phys. 247 (1999) 1 (special issue on single-molecule spectroscopy).

[32] T. Basché, W. Moerner, M. Orrit, U.P. Wild, Single Molecule Optical Imaging and Spectroscopy, VCH, Weinheim, 1996.

[33] Ph. Tamarat, A. Maali, B. Louni, M. Orrit, J. Phys. Chem. A 104 (2000) 1.

[34] W.E. Moerner, M. Orrit, Science 283 (1999) 1670.

[35] B. Kozankiewicz, J. Bernard, M. Orrit, J. Chem. Phys. 101 (1994) 9377.

[36] E. Barkai, R. Silbey, G. Zumofen, J. Chem. Phys. 113 (2000) 5853.

[37] E. Geva, J.L. Skinner, J. Phys. Chem. B 101 (1997) 8920.

[38] E. Geva, J.L. Skinner, Chem. Phys. Lett. 287 (1998) 125.

[39] T. Reinot, W.-H. Kim, J.M. Hayes, G.J. Small, J. Chem. Phys. 104 (1996) 793.

[40] T. Joo, Y. Jia, G.R. Fleming, J. Chem. Phys. 102 (1995) 4063.

[41] W.P. de Boeij, M.S. Pshenichnikov, D.A. Wiersma, Chem. Phys. Lett. 238 (1995) 1.

[42] M.S. Pshenichnikov, K. Duppen, D.A. Wiersma, Phys. Rev. Lett. 74 (1995) 674.

[43] P. Vöhringer, D.C. Arnett, T.-S. Yang, N.F. Scherer, Chem. Phys. Lett. 237 (1995) 387.

[44] P. Vöhringer, D.C. Arnett, R.A. Westervelt, M.J. Feldstein, N.F. Scherer, J. Chem. Phys. 102 (1995) 4027.

[45] M. Toutounji, G.J. Small, S. Mukamel, J. Chem. Phys. 109 (1998) 7949.

[46] M. Lax, J. Chem. Phys. 20 (1952) 1752.

[47] R. Kubo, J. Phys. Soc. Jpn. 9 (1954) 935;

D. terHaar (Ed.), Fluctuation, Relaxation and Resonance in Magnetic Systems: Scottish Universities' Summer School 1961, Oliver \& Boyd, Edinburgh and London, 1962, p. 23.

[48] F. Duschinsky, Acta Physicochem. URSS 7 (1937) 551.

[49] G.J. Small, J. Chem. Phys. 54 (1971) 3300.

[50] Y. Zhao, R.S. Knox, J. Phys. Chem. A 104 (2000) 7751. 